\title{
Características clínico-patológicas y sobrevida de las pacientes con cáncer de mama atendidas en el Instituto Médico de Alta Tecnología (IMAT) (Montería)
}

Título corto: Características de cáncer de mama en IMAT (Montería).

Clinical pathological characteristics and survival of patients with breast cancer attended at IMAT (Montería)

Short title: Characteristics of breast cancer in IMAT (Montería)

- José Lobatón', Manuel González', Fernando Oviedo', Marco Torregroza', Sandra Aruachán²

Servicio de Hematooncología, IMAT Oncomédica.

Unidad de Investigaciones y Estudios Clínicos, IMAT Oncomédica.

\section{Resumen}

El cáncer de mama es un problema de salud pública que amerita especial atención por su incidencia y por su impacto social profundo, ya que afecta a la mujer, eje fundamental de la familia moderna.

Objetivo: describir características clínico-patológicas y la sobrevida global de las pacientes con cáncer de mama atendidas en el IMAT Oncomédica.

Métodos: estudio observacional descriptivo retrospectivo de 506 pacientes con cáncer de mama, donde se analizaron variables socioeconómicas, histológicas, expresión de receptores hormonales y HER2, tratamientos y recaídas.

Resultados: el promedio de edad al momento del diagnóstico fue 54 años. El subtipo histológico predominante fue el ductal y la mayoría de las pacientes presentaron una enfermedad temprana. La tasa de sobrevida global a cinco años en todas las pacientes del estudio fue del $77,6 \%$. La tasa de respuesta patológica completa a la quimioterapia neoadyuvante fue del $11 \%$ en todas las pacientes. La tasa de sobrevida global a seis años en pacientes que recibieron quimioterapia neoadyuvante fue mayor en las que lograron respuesta patológica completa $(74,4 \%)$ versus las que no la obtuvieron $(62,1 \%)(p=0,044)$.

Conclusiones: esta investigación realizada en el IMAT, centro oncológico de referencia para el departamento de Córdoba, es el primer estudio que permite conocer las características clínicas y patológicas de las pacientes con cáncer de mama en esta región, con resultados de sobrevida global similares a países latinoamericanos y cercanos a las cifras de Europa y los Estados Unidos.

Palabras clave (DeCS): neoplasias de la mama, Colombia, enfermedades de la mama, terapia neoadyuvante, sobrevida.

\begin{abstract}
Breast cancer is a public health problem that deserves special attention because of its incidence and its profound social impact because it affects women, the fundamental axis of the modern family.

Objective: The aim of this study was to describe clinical-pathological characteristics and survival rate of patients with breast cancer treated at IMAT Oncomédica.

Methods: Descriptive, retrospective observational study of 506 patients with breast cancer, where socioeconomic, histological variables, hormone receptor and HER2 expression, treatments and relapses were analyzed

Results: The mean age at diagnosis was 54 years. The predominant histological subtype was ductal and most patients had an early disease. The 5 -year overall survival rate in all patients in the study was $77.6 \%$. The pathological response rate to neoadjuvant
\end{abstract}


chemotherapy was $11 \%$ in all patients. The 6 -year overall survival rate in patients receiving neoadjuvant chemotherapy was greater in those who achieved complete pathologic response (74.4\%) versus those who did not achieve complete pathologic response $(62.1 \%)(p=0.044)$.

Conclusions: This research was carried out at IMAT, a cancer reference center for Córdoba. This is the first study to determine the clinical and pathological characteristics of people with breast cancer in this region, with results of overall survival rate similar to Latin American countries and close to the rates of Europe and the United States.

Key words (MeSH): breast, breast neoplasms, survival, survival rate, neoadjuvant therapy.

\section{Introducción}

El cáncer de mama es el tumor maligno más frecuente de la mujer con un aproximado de 1,67 millones de casos nuevos diagnosticados mundialmente en el año 2012, lo cual constituye el 25\% de todos los cánceres. Latinoamérica presenta el mismo comportamiento, con tendencia al aumento de los casos diagnosticados; para el año 2012 se presentaron 115.881 casos de cáncer de mama, con una tasa de incidencia ajustada estimada de 52,2/100.000 mujeres y una tasa de mortalidad de 14/100.000 mujeres'.

En Colombia, las estadísticas de cáncer del año 2012 muestran al cáncer de mama como el más frecuente en la mujer, con 8.686 casos nuevos anuales y una incidencia de 23,4 casos por 100.000 mujeres, con una mortalidad de 10,8 casos por 100.000 mujeres'.

Los factores de riesgo inherentes al paciente para el desarrollo de cáncer de mama son: género, envejecimiento, susceptibilidad genética, antecedentes familiares de cáncer de mama, raza, edad de la menarquia, paridad, terapia de reemplazo hormonal y, en menor proporción, el consumo de alcohol, alimentos grasos y la radiación ionizante ${ }^{2}$.

Aunque se cuenta con diferentes tratamientos para el cáncer de mama, existen factores relacionados con él que afectan la respuesta al tratamiento e inciden en el pronóstico del paciente. Estos factores son: grado del tumor, tipo histológico, tamaño, invasión vascular o linfática, compromiso de ganglios axilares, presencia de receptores hormonales para estrógeno y progesterona (ER/PR), expresión del receptor 2 del factor de crecimiento epidérmico (HER2) y la proteína nuclear Ki67, la cual es un marcador de proliferación celular (Ki67)3,4.

\section{Métodos}

Se realizó un estudio descriptivo retrospectivo con pacientes de cáncer de mama atendidos en el Instituto Médico de Alta Tecnología en el período comprendido entre enero de 2008 y junio de 2013, con un período mínimo de seguimiento de 12 meses. Se excluyeron pacientes que presentaron otro diagnóstico de neoplasia maligna además de cáncer de mama, que tenían un segundo cáncer de mama primario sincrónico, pacientes con historias clínicas con información incompleta y pacientes de género masculino con diagnóstico de carcinoma de mama. Para el tratamiento de los datos producidos por la plantilla de seguimiento, se utilizó el paquete Microsoft $\circledast$ Excel v2008 (versión empresarial), al igual que para las medidas de tendencia central y dispersión, y el análisis de supervivencia mediante la prueba Kaplan-Meier se efectuó con el software estadístico R.

\section{Resultados}

Se revisaron 667 historias clínicas con diagnóstico de cáncer de mama atendidas en el Instituto Médico de Alta Tecnología, en Montería, entre enero de 2008 a junio de 2013. Se descartaron 159 pacientes (24\%) según los criterios de exclusión. Finalmente, se incluyeron en el estudio 506 pacientes. El promedio de edad de las pacientes al momento del diagnóstico fue 54 años con una desviación estándar de 12,7 años. El grupo de edad más afectado fue de 50 a 59 años, donde se ubicaron el $30 \%$ de las pacientes, y el menos comprometido fueron las pacientes menores de 40 años con un $11 \%$, como se observa en la tabla 1.

El subtipo histológico predominante fue el ductal, que afectó al $91 \%$ de las pacientes; el $7 \%$ de los casos correspondió al subtipo lobulillar; el $2 \%$ de las pacientes tenían otro tipo de diagnóstico histológico; y en solo menos del $1 \%$ de las pacientes fue desconocido, como se refleja en la tabla 1.

La mayoría de las pacientes tenían un carcinoma de mama moderadamente diferenciado (grado histológico II), que corresponde al 60\%; los grados histológicos I y III representaron el $18 \%$ y $19 \%$ de las pacientes, respectivamente; y el grado fue desconocido en el $3 \%$ de los casos. 
En relación con el perfil biológico de las pacientes basado en los receptores hormonales y la sobreexpresión o amplificación del HER2, se evidenció que la mayoría de las pacientes presentaron receptores hormonales positivos y HER2 negativo. Los receptores de estrógenos y progesterona fueron positivos en el $65 \%$ y $57 \%$ de las pacientes, respectivamente. La sobreexpresión o amplificación del HER2 fue positiva en el $20 \%$ de las pacientes (ver tabla 2). En la mayoría de las pacientes, el índice de proliferación Ki67 fue desconocido (86\%).

Tabla 1. Características clínico-patológicas de la población

\begin{tabular}{|l|c|}
\multicolumn{1}{|c|}{$\begin{array}{c}\text { Características } \\
\text { clínico-patológicas }\end{array}$} & n (\%) \\
\hline Grupo etario & $55(11)$ \\
\hline$<40$ años & $131(26)$ \\
\hline $40-49$ años & $154(30)$ \\
\hline $50-59$ años & $102(20)$ \\
\hline $60-69$ años & $64(13)$ \\
\hline 70 años & \multicolumn{1}{|c|}{} \\
\hline Subtipo histológico & $459(91)$ \\
\hline Ductal & $34(7)$ \\
\hline Lobulillar & $12(2)$ \\
\hline Otros & \\
\hline Grado histológico & $90(18)$ \\
\hline I & $302(60)$ \\
\hline II & $98(18)$ \\
\hline III & $16(3)$ \\
\hline Desconocido & \\
\hline Estado HER2 & $99(20)$ \\
\hline Positivo & $368(72,7)$ \\
\hline Negativo & $38(7)$ \\
\hline Desconocido & \\
\hline
\end{tabular}

Anualmente se diagnostican y son tratados entre 60 y 104 casos nuevos de cáncer de mama en nuestra institución. Al diagnóstico el $51 \%$ de las pacientes presentaron una enfermedad temprana (estadios I y II), locorregionalmente avanzada (estadio III) en el $41 \%$ de los casos y con metástasis a distancia (estadio IV) en el $5 \%$. La distribución de las pacientes por cada uno de los estadios de acuerdo con el sistema de estadificación TNM es presentada en la figura 1.

En las pacientes sin metástasis al diagnóstico de la enfermedad (483 pacientes), se realizó quimioterapia neoadyuvante al $53 \%$ de ellas, obteniendo respuesta patológica completa en el $11 \%$ de las pacientes y, según el perfil biológico, se obtuvo respuesta patológica completa en el 38\% de las pacientes con sobreexpresión de HER2 y en el $15,8 \%$ de las pacientes triple negativo

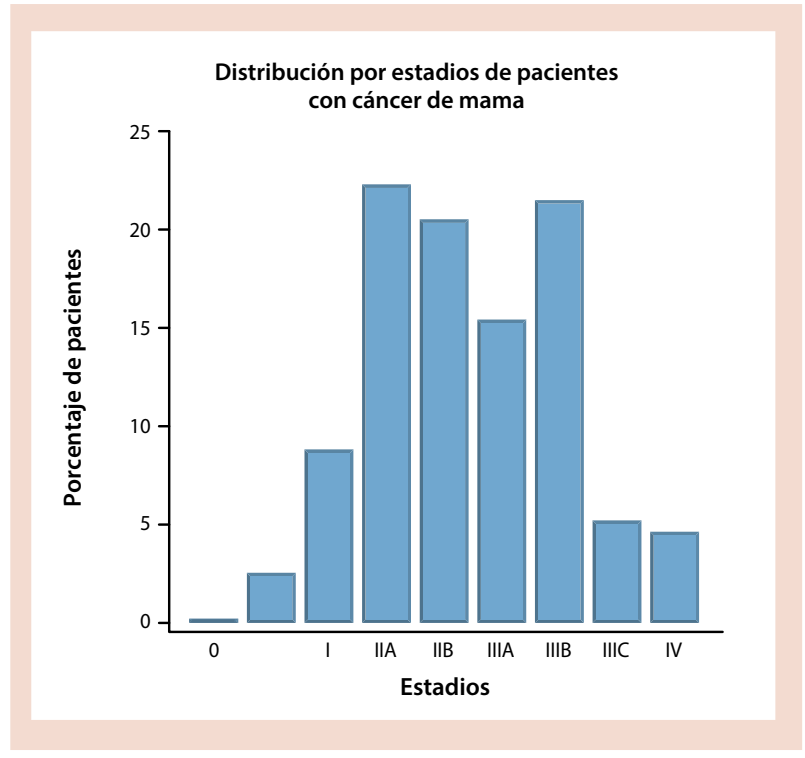

Figura 1. Distribución de las pacientes por estadios TNM.

(receptores hormonales y HER2 negativos). Posterior a la quimioterapia neoadyuvante, el tratamiento quirúrgico practicado fue mastectomía radical modificada y cuadrantectomía en el $72 \%$ y $28 \%$ de los casos, respectivamente.

Los esquemas de quimioterapia más utilizados, tanto en el escenario neoadyuvante como adyuvante, incluían antraciclinas y taxanos usados secuencialmente. En neoadyuvancia, el esquema de quimioterapia más usado fue doxorrubicina más ciclofosfamida (esquema AC) en el 57\%, mientras que el esquema secuencial AC seguido de paclitaxel fue empleado en el $23 \%$ de las pacientes. El $86 \%$ de las pacientes HER2 positivo y con enfermedad no metastásica al diagnóstico recibieron trastuzumab adyuvante. La hormonoterapia adyuvante más utilizada fue tamoxifen en el $70 \%$.

Las pacientes presentaron recaída de la enfermedad en el $20 \%$ de los casos y las recaídas a distancia fueron las más frecuentes (88\%). Las tasas de recaída en las pacientes HER2 positivo y en las triple negativo fueron del $31,3 \%$ y $23,1 \%$, respectivamente. Los órganos más frecuentemente comprometidos por metástasis fueron pulmones y huesos con afectación del $39 \%$ en cada uno de ellos. Para el tratamiento de la enfermedad metastásica, se emplearon múltiples agentes de quimioterapia y hormonoterapia.

La tasa de sobrevida global a cinco años en todas las pacientes del estudio fue del $77,6 \%$ (figura 2) y, de acuerdo con el perfil biológico, los resultados de sobrevida global a cinco años se 


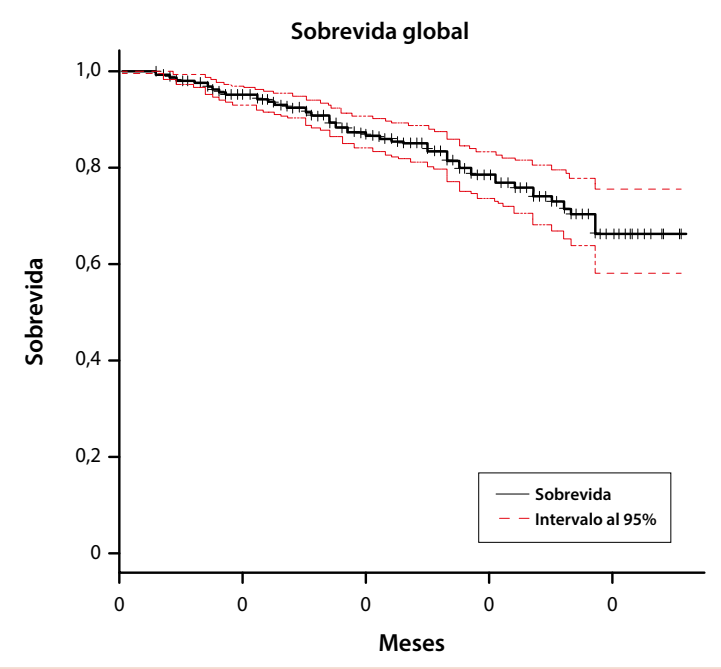

Figura 2. Sobrevida global.

observan en la figura 3. Al momento del último control realizado, el $79 \%$ de las pacientes estaban vivas sin enfermedad.

La tasa de sobrevida global a seis años en pacientes que recibieron quimioterapia neoadyuvante fue mayor en las que lograron respuesta patológica completa $(74,4 \%)$ versus las que no la obtuvieron $(62,1 \%)$ $(p=0,044)$.

\section{Discusión}

El promedio de edad de las pacientes al momento del diagnóstico es 54 años, siendo el grupo etario más afectado las de 40 a 59 años, que constituyen el $56 \%$ de ellas, resultados similares a los reportados por el Instituto Nacional de Cancerología en la publicación de Incidencia, mortalidad y prevalencia de cáncer en Colombia 2007-2011, en donde la incidencia estimada por cáncer de mama por grupos de edad en mujeres en el departamento de Córdoba entre 2007 a 2011 describe que el grupo más comprometido corresponde a las pacientes entre $45 \mathrm{y}$ 64 años de edad 5 .

En este análisis se evidenció que la mayoría de las pacientes presentan receptores hormonales positivos y HER2 negativo; los receptores de estrógenos y progesterona son positivos en el $65 \%$ y $57 \%$ de las pacientes, respectivamente, resultados similares a lo descrito en un estudio de 5.497 casos que reportó positividad para receptores de estrógenos y progesterona del $75 \%$ y $55 \%$, respectivamente 6 . El $20 \%$ de las pacientes presentan sobreexpresión del HER2, resultados concordantes con los reportados en la literatura mundial con una positivad del HER2 entre el $15 \%$ y $25 \%$.

Solo un $5 \%$ de las pacientes debutan con enfermedad metastásica al diagnóstico, mientras que el $51 \%$ de ellas presentan una enfermedad temprana y locorregionalmente avanzada en el $41 \%$ de los casos, distribución concordante con un estudio de 232 pacientes realizado en el Hospital Universitario San Ignacio de Bogotá, que reportó que los estadios IV al diagnóstico fueron del 7\% y los casos locorregionalmente avanzados del $42,3 \%{ }^{8}$.

La respuesta patológica completa obtenida posterior a la quimioterapia neoadyuvante fue del $11 \%$ en todas las pacientes que recibieron esta opción terapéutica; la respuesta patológica completa en las pacientes HER2 positivo fue del $38 \%$, resultado consistente con lo descrito con esquemas de neoadyuvancia basados en trastuzumab con respuestas patológicas completas que oscilan entre el $29 \%$ y $53 \%$. Las respuestas patológicas completas en la pacientes triple negativo fueron del $15,8 \%$, resultado menor a lo establecido con esquemas de quimioterapia neoadyuvante que incluyen una antraciclina y un taxano secuencialmente con respuestas entre el $22 \%$ y $40 \%$; esto podría ser explicado en parte porque en el presente estudio el esquema de quimioterapia neoadyuvante secuencial AC seguido de paclitaxel fue empleado solo en el $23 \%$ de las pacientes ${ }^{9}$.

Hubo recaída de la enfermedad en el $20 \%$ de las pacientes y las recaídas a distancia fueron las más frecuentes, representando el $88 \%$ de los casos. Las pacientes triple negativo tuvieron una tasa de recaída del 23,1\%, que es menor a lo evidenciado en un estudio hecho en la clínica Oncocare de Bogotá, que fue del $39,9 \%$. Los órganos más frecuentemente comprometidos por metástasis fueron los pulmones y los huesos con afectación del 39\% de los casos en cada uno de ellos, resultados similares a lo publicado a nivel mundial y a lo reportado por una investigación colombiana, la cual evidenció que los sitios más afectados por metástasis fueron huesos (35\%), pulmones (17\%) e hígado $(11 \%)^{8}$.

La tasa de sobrevida global a cinco años en todas las pacientes del estudio fue el $77,6 \%$ menor a lo reportado en países desarrollados, como los Estados Unidos (90\%) y Reino Unido (86\%), pero comparable a lo evidenciado 
en países latinoamericanos, como Brasil (58\%), México (58\%), Perú (67\%), Costa Rica (70\%) y Chile (76\%). Según el perfil biológico, la sobrevida global a cinco años fue mayor en pacientes con receptores hormonales positivos $(83,4 \%)$, seguido por mujeres HER2 positivo (70.9\%) y, por último, las pacientes triple negativo $(60,8 \%)$, resultados también similares a lo descrito en la literatura mundial ${ }^{10}$.

En el presente estudio se encontró como factor pronóstico de sobrevida global obtener una respuesta patológica completa a la quimioterapia neoadyuvante; la tasa de sobrevida global a seis años fue mayor en las pacientes que lograron respuesta patológica completa $(74,4 \%)$ versus las que no la obtuvieron $(62,1 \%)(p=0,044)$.

Estos resultados están dentro del rango de lo reportado por otros estudios para las pacientes que obtienen respuesta patológica completa con tasas de sobrevida global a los cinco años, que varían entre el $53 \%$ y $82 \%{ }^{11}$.

La efectividad del tratamiento del cáncer de mama en la clínica IMAT objetivizada por una tasa de sobrevida global a cinco años del 77,6\%, que consideramos óptima, se debe en gran parte a que en esta institución se realiza un manejo integral de todas las patologías malignas, permitiendo una intervención multidisciplinaria de todas las especialidades médicas y quirúrgicas, servicios clínicos y paraclínicos de apoyo que están involucrados en el diagnóstico, tratamiento y seguimiento del cáncer de mama.

\section{Conclusiones}

El presente estudio realizado en el IMAT, que es el centro oncológico de referencia para el departamento de Córdoba, es el primero que permite conocer las características clínicas y patológicas de las pacientes con cáncer de mama en esta región de Colombia, con resultados de sobrevida global similares a países latinoamericanos y cercanos a las cifras de Europa y los Estados Unidos.

\section{Referencias}

1. Ferlay J, Soerjomataram I, Dikshit R, Eser S, Mathers C. Globocan 2012 v.1.0, Cancer incidence and mortality worldwide: IARC Cancer Base No 11 [internet]. 2013 [citado 2017]. Disponible en: "www.globocan.airc.fr

2. DeVita V, Lawrence T, Rosenberg S. Cancer principles \& practice of clinical oncology. 9th ed. Philadelphia: Wolters Kluwer; 2011.

3. Inwald EC, Klinkhammer-Schalke $M$, Hofstädter F, Zeman F, KoIler M, Gerstenhauer M, et al. Ki-67 is a prognostic parameter in breast cancer patients: results of large population-based cohort of a cancer registry. Breast Cancer Res Treat 2013;139(2):539-52.

4. Coronato S, Laguens G, Spinelli O, Girolamo W. Marcadores tumorales en cáncer de mama. Medicina 2002;62:73-82.

5. Pardo C, Cendales R. Incidencia, mortalidad y prevalencia de cáncer en Colombia 2007-2011. $1^{a}$ ed. Instituto Nacional de Cancerología; 2012.

6. Nadji M, Gómez-Fernández C, Ganjei-Azar P, Morales A. Immunohistochemistry of estrogen and progesterone receptors reconsidered: experience with 5,993 breast cancers. Am J Clin Pathol 2005;123(1):21-7.

7. Nguyen $\mathrm{PL}$, Taghian AG, Katz MS, Niemierko A, Abi Raad RF, Boon WL. Breast cancer subtype approximated by estrogen receptor, progesterone receptor, and HER-2 is associated with local and distant recurrence after breast-conserving therapy. J Clin Oncol 2008;26(14):2373-8.

8. Angarita F, Acuña $S$, Torregrosa $L$, Tawil M, Ruiz A. Presentación inicial de las pacientes con diagnóstico de cáncer de seno en el Centro Javeriano de Oncología, Hospital Universitario San Ignacio. Revista Colombiana de Cirugía 2010;25(1):19-26.

9. Martin-Castillo B, Lopez-Bonet E, Cuyas E, Viñas G, Pernas S, Dorca J, et al. Cancer stem cell-driven efficacy of trastuzumab (Herceptin): towards a reclassification of clinically HER2-positive breast carcinomas. Oncotarget 2015;6(32):32317-38.

10. Jemal A, Bray F, Center MM, Ferlay J, Ward E, Forman D. Global cancer statistics. CA Cancer J Clin 2011;61(2):69-90.

11. Kaufmann $M$, Hortobagyi $G$, Goldhirsch A, Scholl S, Makris $A$, Valagussa $P$, et al. Recommendations from an international expert panel on the use of neoadjuvant (primary) systemic treatment of operable breast cancer: an update. J Clin Oncol 2006;24(12):1940-9 